\title{
Is The Computer The Medium And The Message? A Comparison Of Student VB Programming Performance In Three Delivery Modes
}

Wayne Haga, Ph.D, (E-mail: haga@mscd.edu), Metropolitan State College of Denver Kathryn A. Marold, Ph.D., (Email: maroldk@mscd.edu), Metropolitan State College of Denver

\begin{abstract}
As a third stage of research on delivery of a popular programming course at Metropolitan State College of Denver, the authors gathered data from an online section of CIS Application Programming with Visual Basic, two interactive classroom deliveries in campus smart classrooms, and in two traditional lecture-based sections of the course. The authors had previously completed research which showed significant differences in student ability to apply concepts learned in online sections and classroom sections of the Visual Basic programming class. The addition of interactive classrooms on campus allowed them to reexamine the relationship between performance and style of delivery. The three modes of delivery--1) Web 2) Interactive Classroom and 3) Traditional Classroom--provided a rich source of data on student ability to apply theory. Data was gathered on a lab exam, a three phase semester-long programming project, and essay/coding problems on three in-class exams.
\end{abstract}

Data from the five sections of the Visual Basic course were analyzed. Although the results were not as conclusive as the authors had hoped, an analysis of variance showed significant differences in one of the three measurements of students ability to apply theory - the programming project. A Fisher's pairwise comparison indicated that the Internet students performed significantly lower than students receiving the traditional classroom or interactive instruction method. The attrition rate for the Internet section was more than twice that of the other modes of delivery. Although the Interactive Classroom mode of delivery was not found to be significantly different, students did score slightly higher. All sections of the course used the same syllabus and assignments; the instructors used common examinations and rubrics for grading student assignments.

The authors conclude that an interactive environment, within an instructor-led "smart classroom ", is preferable to Web based delivery, and perhaps the traditional lecture-based method of delivering programming classes. This style of delivery is more apt to produce a student who can apply theory and produce working code. As evidenced by performance on projects and subjective tests, students who participated in the interactive classroom sections were better able to apply programming theory to new situations. The authors caution that while traditional classroom delivery methods are well-established, Web courses and the interactive classrooms are still in their infancy. Further research is needed to verify their conclusions.

Readers with comments or questions are encouraged to contact the authors via email. 


\section{Introduction}

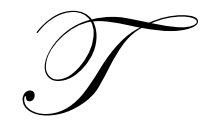

he 21 st century is upon us and the traditional methods of delivering college curricula are giving way to a new model. We know that Web courses are here to stay. The last decade of the 20th century saw a mad rush to put college courses, and entire college curricula, online so that students could earn an entire degree without setting foot on a campus. Time and again, no significant differences were found in final grades between Web and classroom delivery of courses. The general consensus was that Web courses were working, and that colleges were justified in forging ahead with plans to put entire curricula online. After the first rush of research on differences among Web courses and classroom courses, several educators found serious flaws in the first conclusions about the success of online education. In 2001 our research on performance and achievement between the two scenarios found performance to be significantly lacking in students who were taking the Web section of a programming course (Marold, 2002.) We wondered if the interactive environment of a "smart classroom" could somehow bring the best of each mode and compensate for the inadequacies of online instruction.

The first courses to be put online in Information Systems departments were generally the lower level elective courses, and courses which were survey or retention-oriented courses. That is, the material of the first computer literacy course, the first information systems survey course, introduction to the Internet, productivity software courses, and self-paced courses was simpler to port to the Web environment. An average knowledge of instructional design and a crash course in Web page editing with Front Page ${ }^{\circledR}$, or even WebCT ${ }^{\circledR}$ or other course authoring software was enough to move these "to-do list" type of courses to the Web. The actual content of the course was not within the Web pages of the course, but in textbooks and assigned readings listed on the course Web pages. Therefore, the student was actually doing the "learning" apart from the Web pages of the course, usually by doing tutorials or homework assignments. It was time to re-examine all three available delivery modes, and determine if one mode was superior. Not the least important was the question, "Does the use of computer technology to learn computer programming make a difference?" Is the medium and the message finally melded so closely that without a computer, learning programming theory is hampered?

\section{Research questions}

We had recently offered sections of CMS 3145 Business Application Development with Visual Basic on the Web, following the other department courses that had been developed for Internet delivery. The addition of "smart classrooms" (lecture halls where every student has a computer and the Instructor system is projected on highend equipment for everyone to follow along) provided yet another scenario to test. In the first semesters of the online course, we noticed numerous egregious errors by online students in application of theory on tests, homework, and projects. In some cases there was a complete lack of understanding about the big picture. Errors in work demonstrated lack of understanding of crucial topics on the part of Internet students. This was documented in our previous study (Haga, 2001.) Would this be the case with students in the smart classrooms as well?

We decided to re-examine the question of performance within non-traditional modes of delivery. Perhaps the technology was getting in the way of mastering programming theory? Or perhaps what was lacking in the asynchronous and separated environment of the Web course would be counteracted by physically meeting with students in a smart classroom. A robust analysis of learning in the three different modes could point out serious flaws in using technology to such a great extent in our CIS courses. Lack of true understanding of concepts appeared to be a problem, but it was not certain how widespread it was, nor what was contributing to it. We previously found that Web courses above the survey or intro level (ones where content centered on familiarizing and identifying) that involved problem solving, thinking, analyzing and generally applying theory learned were not working as well (Marold, 2001.) Would smart classroom delivery make a difference? We suspected from observation and grading of assignments and tests that there may be significant differences in student ability to apply IS theory in all three delivery modes. We wanted to know if this was true, and if it was, what could be done to improve the delivery of our upper level Information Systems courses. 


\section{Literature Review}

A wealth of research has already been done on final grades, design of Web courses and delivery of hybrid courses (Presby, 2001.) Research has reported the advantages and disadvantages of Web courses, for both students and faculty (Mawhinney, 1998.) The social aspects of online student behavior has been researched (McCloskey, 1998.) McCloskey found that student satisfaction with the Internet is growing. Communication habits of instructor and students were found to be greater in online courses (McGinnis, 1998.) Burgstahler found that students participate more in class discussions when the course is delivered electronically than they would in a traditional class (1997.) Despite some obvious flaws, Internet courses were not going away. Student demand for complete degrees and certificates of training was found to be increasing significantly (Nixon, 1998.) Kroder reported that 8 out of 10 students who responded to a survey of Web course satisfaction said they would take another Internet-based course even though it took more time than a classroom course (1998.)

Differences in final grades between Internet students and classroom students have been found not to be statistically significant (Schulman, 1999; Mawhinney, 1998; Bowman, 1995; McCloskey, 1998.) The credibility of courses completed online as opposed to the classroom has been analyzed (Moreno, 2000.) Research on the levels of computer literacy by students taking Web courses showed that most students were at a competent level on the Bodker scale, but not at the novice or proficient level. [Authors' note: The scale developed in 1991 rates individuals on a five-point scale, from Novice, to Beginner, to Competent, to Proficient, to Expert (Bodker, 1991.)] What is more, by the time students took a 3000 level course, $63 \%$ of them had taken an Internet course before (Marold, 2000.) Web literacy and computer literacy in general did not seem to be a barrier to taking an upper level course online. This is an indication that the delivery environment was not an impediment to the course mastery. As Shank has noted, the traditional classroom course has been revolutionized by the saturation of the computer and the Internet within our lives (2001.)

The attrition rates and failure rates for online courses, and all distance education courses in general, have always been higher than in the classroom (Frankola, 2001; Larsen, 1998.) Terry found that the attrition rates for online MBA courses not only were higher than in the classroom, but as the courses became more analytical and theoretical, the attrition rates increased (Terry, 2001.) The attrition rate in Quantitative Analysis was 33\%, and in Statistical Methods in Business was an astounding 43\%. The Finance courses also had a higher than normal attrition rate $(24 \%$.) The natural conclusion was that courses requiring extensive mathematics and problem solving were more difficult to convert to an Internet format. The course that was the subject of this study requires a higher degree of analytical and problem solving ability; its perquisite is a business programming logic course. As expected, in this study, the interactive classroom had the lowest attrition rate with only 6 of $44(16 \%)$ of the students not completing the course. The attrition rate for the traditional classroom was slightly higher at 10 of 51 (20\%), followed by a much higher rate for online students at 10 of 21 (47\%). We decided to investigate the differences in ability of Internet, smart classroom, and traditional classroom students to apply the theory contained in the programming course.

\section{Research Plan}

We gathered data from the three groups of online, interactive, and classroom sections of one programming course. The Visual Basic course had been Web-delivered for two years. Grades from projects, application portions of tests, and a hands-on lab exam were gathered and analyzed. The plan was to then use a one-way ANOVA (analysis of variance) model to compare the three delivery modes separately for projects, application of theory exams questions, and the lab test, to see if there were differences in student ability to apply concepts learned, and if differences could be attributed to delivery mode. The research questions then could be phrased as follows: 
Null Hypothesis 1: $\quad$ There is not a significant difference between the three delivery modes as evidenced by scores on programming projects.

Alternate Hypothesis 1: At least one delivery method is significantly different as evidenced by grades on programming projects.

Null Hypothesis 2: $\quad$ There is not a significant difference between the three delivery modes as evidenced by scores on applied exams.

Alternate Hypothesis 2: At least one delivery method is significantly different as evidenced by grades on programming projects applied exams.

Null Hypothesis 3: $\quad$ There is not a significant difference between the three delivery modes as evidenced by scores on a hands-on lab exam.

Alternate Hypothesis 3: At least one delivery method is significantly different as evidenced by grades on a handson lab exam.

\section{Methodology}

We developed a plan to collect data from student scores on applied portions required of all students in the Business Application Development with Visual Basic course. The data were entered and analyzed with the Minitab software program. In all three delivery modes of the Visual Basic programming course, the student assignment was to submit three phases of a semester-long project. Each phase covered four or five chapters of programming concepts. Students were required to apply concepts from each chapter by designing, coding, and debugging functions and subroutines to process forms. Students were also required to design the form layouts, choosing appropriate controls. Three exams were given, with each exam covering the same material as the three phases of the project. Part II of each of these exams consisted of approximately five essay/coding problems. A hands-on lab exam was also administered, requiring students to design, code and debug a small project within an hour. The average of the three phases of the project was calculated, as was the average of the applied portions of the three exam scores. For the purpose of this analysis, it was decided that students would be graded only on work attempted, assuming at least two of the three scores were available. Thus the average for a project score or exam score could be based on either two or three scores. (No average was calculated for students completing only one of the three scores.)

\section{Analysis}

A one-way ANOVA model was used to compare the difference between classroom, interactive, and Internet students separately on each of the three evaluation criteria. If the ANOVA showed a significant difference between evaluation methods, Fisher's multiple comparison method was used to identify which pairs were different.

\section{Results}

One-way ANOVA results are shown in the following three tables. Results were not as conclusive as we had hoped, but they do indicate a difference in delivery modes. Table I shows the results of student performance on projects for the three delivery methods. It was found that there was a significant difference between delivery methods $(\mathrm{F}=5.24, \mathrm{p}=.007)$. Fisher's pairwise comparison (alpha=.05) indicated there was a significant difference between the mean score on projects between the Interactive classroom (84.1\%) and the Internet class (68.0\%). It also indicated there was a significant difference between the regular Classroom (81.6\%) and the Internet class $(68.0 \%)$. The difference between the Interactive and regular Classroom methods was not found to be significant, although the Interactive students did score 2.5 percentage points higher. Also notable is the much higher standard deviation of students in the Internet course (Internet - 27.6, Classroom - 13.6, Interactive - 12.2) indicating a much wider range of performance. The instructor reported that students in the Internet section basically fell into two groups - those who performed well, and those who failed to meet minimum standards, with a wide spread in the latter group. 
Table I: CMS 3145 Project Results

One-way Analysis of Variance

\begin{tabular}{|l|l|l|l|l|l|}
\hline Source & DF & SS & MS & F & P \\
\hline Delivery & 2 & 2572 & 1286 & 5.24 & 0.007 \\
Error & 92 & 22583 & 245 & & \\
Total & 94 & 25155 & & & \\
\hline
\end{tabular}

\begin{tabular}{|l|l|l|l|}
\hline Level & $\mathrm{N}$ & Mean & StDev \\
\hline Interactive & 37 & 84.14 & 12.22 \\
Classroom & 45 & 81.62 & 13.56 \\
Internet & 13 & 68.00 & 27.57 \\
\hline
\end{tabular}

Fisher's Pairwise Comparison

\begin{tabular}{|l|l|l|}
\hline $\begin{array}{l}\text { Family error rate } \\
\text { Individual error rate } \\
\text { Critical value }\end{array}$ & .121 \\
\multicolumn{2}{|l|}{} & .05 \\
\hline \multicolumn{2}{|l|}{ Interval for (column level mean) - (row level mean) } \\
\hline & Interactive & Classroom \\
\hline Classroom & -4.39 & \\
& 9.42 & 3.82 \\
\hline Internet & 6.10 & 23.42 \\
& 26.17 & \\
\hline
\end{tabular}

Table II: CMS 3145 Exam Results One-way Analysis of Variance

\begin{tabular}{|l|l|l|l|l|l|}
\hline Source & DF & SS & MS & F & P \\
\hline Delivery & 2 & 141 & 71 & 0.28 & .757 \\
Error & 91 & 23018 & 253 & & \\
Total & 93 & 23159 & & & \\
\hline
\end{tabular}

\begin{tabular}{|l|l|l|l|}
\hline Level & $\mathrm{N}$ & Mean & StDev \\
\hline Interactive & 37 & 70.91 & 14.61 \\
Classroom & 45 & 68.32 & 14.88 \\
Internet & 12 & 70.17 & 22.56 \\
\hline
\end{tabular}

Table III: CMS 3145 Lab Exam Results

One-way Analysis of Variance

\begin{tabular}{|l|l|l|l|l|l|}
\hline Source & DF & SS & MS & F & P \\
\hline Deli- & 2 & 539 & 270 & 1.44 & 0.242 \\
very & 99 & 18533 & 187 & & \\
Error \\
Total
\end{tabular}

\begin{tabular}{|l|l|l|l|}
\hline Level & $\mathrm{N}$ & Mean & StDev \\
\hline Interactive & 42 & 81.64 & 11.64 \\
Classroom & 47 & 78.09 & 14.80 \\
Internet & 13 & 84.54 & 15.54 \\
\hline
\end{tabular}

Table II shows the results of student performance on essay/coding problems on inclass exams for the three delivery methods. It was found that there was not a significant difference between delivery methods $(\mathrm{F}=.28$, $\mathrm{p}=.757)$. The students in the Interactive course did score slightly higher (70.9) than students in the traditional Classroom (68.3) and Internet class (70.2). The standard deviation was again much higher for the Internet class (Internet - 22.6, Classroom 14.9 , Interactive - 14.6).

Table III shows the results of student performance on the hands-on lab exam for the three delivery methods. It was again found that there was not a significant difference between delivery methods ( $\mathrm{F}=1.44, \mathrm{p}=.242)$. The Internet students slightly outperformed the other two groups (Internet - 84.6, Interactive 81.6, Classroom - 78.1) It is notable, however, that while Internet students" "lab" test was taken in a timed environment during a required calendar, where they uploaded their finished program to the instructor's Workspace, they were in an unproctored environment, in the comfort of their own homes or work settings.

\section{Discussion}

The results point towards what we have suspected, that Internet students are having trouble applying the concepts of programming they are attempting to learn without the shared learning environment or the guidance of an instructor in a classroom. Interactive classes appear to do best, and the traditional classroom students exceed the Internet section on projects. However, the Internet section of the class was small, due to limited enrollment and the aforementioned high attrition rate. As noted earlier, we previously conducted a study that gave mixed results in the comparison of online and classroom students in the Visual Basic course (Haga, 2001.) The fact that each of these observations for the projects and written exams are based not on one score, but at least two and usually three scores should lend credibility to the results, especially since each score is based on numerous questions or sections of programming code. 
The analysis of the data gathered on the five CIS classes researched indicates that at the very least, such application of theory classes offered online merit more in-depth research and some serious attention. It is very clear that demonstration of theoretical knowledge in Internet classes is below that of traditional classes, and interactive delivery within smart classrooms is the best delivery mode within the IS program at this institution. The students who did not attempt essay questions or one of the phases of the project were more numerous in the Internet group. More phases of the project were missing in the Internet section, as well. (Due dates for projects are strictly enforced in all sections of the Visual Basic programming course-whether online or not.) Essay questions that require discussion and demonstration of theory have always been part of the Visual Basic class, but perhaps not of other courses students may have taken on the Web. The data analysis might also show students' expectations for this 3000 level course may have been lower than the instructors' requirements. Accustomed to self-paced syllabus-type courses, Internet students may not have been accustomed to a course where there was more than a list of assignments to turn in at scheduled times and one or two objective tests. Furthermore, students in the interactive class had to be comfortable with the technology to get the most out of the environment. Although it should have been true, some of the time it was not.

\section{Authors' Interpretations and Suggestions for Possible Solutions}

Traditional classroom instructors emphasize important content and encourage application of that content in ways that are not even apparent to the instructor - often through subtle changes in voice or body language that are simply instinctive for effective instructors. Application of theory may be effectively illustrated in the classroom by simple choice of examples or answers given to questions - none of which constitute a conscious effort on the part of the instructor. Simple repetition can be effective in the traditional or interactive classroom, but is difficult to implement online. Since there is no way to ensure that a student in an online course will return to an important concept, the first exposure may well be the only exposure to that particular concept.

Group interaction can be a significant component of the learning process in the classroom setting, but even thoughtful attempts to use online forums, chat sessions and e-mail don't necessarily duplicate classroom group experience in the online environment. Students also get more immediate feedback to questions in the classroom environment, which may result in more and better questioning. The group investigation and discovery often found in the classroom can be much more rewarding. Students feed off of each other's thoughts and comments and find true satisfaction in the joy of recognition as a group. Although active chat sessions and virtual office hours were used in the Web section of the Visual Basic programming course, group discovery at a distance is simply not the same. The cohesiveness and satisfaction of class discovery is not duplicated in a chat room or asynchronous forum. Only a maximum of six or seven students were in the chat session with the instructor at any one time. A high level of interstudent activity was not required for the Web course. The virtual office hours via Chat with Whiteboards were not obligatory; they were a synchronous online equivalent of physical office hours at campus.

\section{Suggestions}

The new model of delivering course content demands new assessment methods and increased interactivity. Web delivered sections of a course could benefit from materials that in some way duplicate the use of examples and questions in the classroom. Application of smaller content elements can be required (using simple worksheets or open-ended questions) before major projects are presented. Frequent interactive elements in course materials could be especially effective. Required Forum postings or Chat sessions had been avoided, since students report that one of the major reasons for taking an online course was the "Any Time Any Place" freedom that Web courses afford. There are plans for more short exercises in the future smart classroom sections of the class. The "to-do list" of pages that directed the online student to a textbook or readings characterized earlier Web courses. It appears this is simply not sufficient for more advanced problem solving, analytical courses. More interactive exercises and presentation of examples of application of theory might be indicated for all delivery modes. All three sections contained slide lectures of theory and links to programming examples, and practice quizzes to prepare for exams. They were largely unused in all three delivery modes. Students and instructors alike must be educated on how to succeed in an interactive environment, and must have practice doing it. This requires time and experience. 


\section{Conclusions}

It is clear that more research on the success of Web-based and interactive "smart" classroom courses needs to be done. We plan to continue to gather and analyze data comparing traditional classroom sections, interactive sections, and Web sections of upper level IS classes. To attempt to remove any differences in the populations in the three groups, a problem solving/logic screening test is administered during the first two weeks of class. Other upper level courses that concentrate on application of theory will also be examined. Additional online versions of departmental CIS courses have recently been developed. Within the next two years an administrative goal is to have all of the required CIS courses online. There will be a much larger sample from many other IS arenas from which to test the hypotheses. We remain committed to finding the most successful mode for delivery of department programming courses. We justifiably remain concerned about the reality of developing critical thinking skills and analytical reasoning online and in interactive classrooms. The realization that we have survived the initial computer-delivered environment moves us to be more committed to completing more in-depth research and better delivery of programming courses, whatever that delivery mode might be.

\section{References}

1. $\quad$ Bodker, S. (1991.) Through the Interface. Hillsdale, NJ. Lawrence Erlbaum Associates.

2. Bowman, B.J. et al. (1995.) Teaching End User Applications with Computer -based Training. Theory and an Empirical Investigation. Journal of End User Computing. 7:2. 12-17.

3. Burgstahler, S. (1997.) Teaching on the Net: what's the Difference? T.H.E. Journal. 24:9. 61-64.

4. Frankola, K. (2001.) The e-Learning Taboo: High Dropout Rates in Online Courses. Syllabus. June 01. 14.

5. Kroder, S.L., J. Suess and D. Sachs. (1998.) Lessons in Launching Web-based Graduate Courses. T.H.E. Journal. 25: 10. 66-69.

6. Larsen, G. and S. Helms. (1998.) The Internet as an Instructional Delivery Vehicle for Higher Education: a Framework for Evaluation. Proceedings of the International Association for Computer Information Systems. Las Vegas, NV. September.

7. Haga, W., K. Marold, and S. Helms. (2001.) Round 2 of Online Learning: Are Web Courses Really Working? Proceedings of the Twenty-ninth Annual Conference of the International Business Schools Computing Association. Providence, R.I. July 17, 2001. 22-33.

8. Marold, K. and W. Haga. (2002.) Measuring Online Students' Ability to Apply Programming Theory: Are Web Courses Really Working? Proceedings of the Western Decision Sciences Institute Annual Meeting. Las Vegas, NV. April 4-6, 2002.

9. Marold, K., G. Larsen, A. Moreno. (2000.) Web-based Learning: Is It Working? Challenges of Information Technology Management in the 21st Century. Idea Group Publishing. Hershey, PA. 350-353.

10. Mawhinney. C. et al. (1998.) "Issues in Putting the Business Curriculum Online. Proceedings of the Western Decision Sciences Institute. Puerto Vallarta, MX. October.

11. McCloskey, D. et al. (1998.) Web-based vs. Traditional Course Deployment: Identifying Differences in User Characteristics and Performance Outcomes. Proceedings of IBSCA Annual Conference. Denver, CO. July 1998.

12. McGinnis, D. et al. (1998.) "Student /Faculty Collaboration for Online Course Development." Proceedings of FACT Symposium Panel Conference. Glenwood Springs, CO. April 23, 1998.

13. Mitchell, E.J. and S.H. Frisbie. (2001.) An Experiment with Student-centered Learning. Syllabus. 30-33. Sept. 2001.

14. Moreno, A., G. Larsen, and K. Marold. (2000.) The Credibility of Online Learning: a Statistical Analysis of IS Course Delivery at Three Levels. Western Decision Sciences Institute. Maui, HI. April.

15. Nixon, M.A and B.R. Leftwich. (1998.) Leading the Transition from the Traditional Classroom to a Distance Learning Environment. T.H.E. Journal. 2: 6. 54-57.

16. Presby, L. (2001.) Increasing Productivity in Course Delivery. T.H.E. Journal. 28: 7. 52-58.

17. Schulman, A., and R.L. Sims. (1999.) Learning in an online format versus an In-class Format: An Experimental Study. T.H.E. Journal. 26: 11. 54-56.

18. Shank, R. (2001.) The Computer isn't the Medium, It's the Message. Communications of the ACM. 44:3. 142-143. 
19. Shank, R. (2001.) Revolutionizing the Traditional Classroom Course. Communications of the ACM. 44: 12. 21-24.

20. Terry, N. (2001.) Assessing Enrollment and Attrition Rates for the Online MBA. T.H.E. Journal. 28: 7. 6468. 